\title{
Supporting Information \\ Toward Analysis of Proteins in Single Cells: A Quantitative Approach Employing Isobaric Tags with MALDI Mass Spectrometry Realized with a Microfluidic Platform
}

\author{
${ }^{1,2}$ Mian Yang, ${ }^{3}$ Randall Nelson, ${ }^{1,2}$ Alexandra Ros* \\ ${ }^{1}$ School of Molecular Sciences, Arizona State University, Tempe, AZ 85287, USA \\ ${ }^{2}$ Center for Applied Structural Discovery, The Biodesign Institute, Arizona State University, \\ Tempe, AZ 85287, USA \\ ${ }^{3}$ Molecular Biomarkers Laboratory, The Biodesign Institute, Arizona State University, Tempe, \\ AZ 85287, USA
}

\section{Supplementary information}

This section contains additional information including the error determination of iTRAQ labeling on Bcl-2 with different labeling ratios, sensitivity of iTRAQ quantification strategy with Bcl-2, sensitivity of Bcl-2 detection with and without affinity capture, and database searching of Bcl-2 digest peaks for Bcl-2 identification. 


\section{Influence of iTRAQ ratios on the quantification of Bcl-2}

The quantification of Bcl-2 on-chip was achieved by using two iTRAQ tags. The MS/MS analysis allows for the detection of the iTRAQ labels whereas the peak area ratio of $114 \mathrm{tag} / 117$ tag is used to determine unknown concentrations of the peptides and thus the corresponding protein. To demonstrate the applicability of the peptide tagging by iTRAQ for Bcl-2, digests were differentially labeled with 114 tag and 117 tag. Accordingly, $2 \mu \mathrm{L}$ of $1.4 \times 10^{-6} \mathrm{M} \mathrm{Bcl-2}$ was mixed with $8 \mu \mathrm{L}$ iTRAQ dissolution buffer, $2 \mu \mathrm{L}$ trypsin solution $(0.1 \mathrm{mg} / \mathrm{mL})$ and $8 \mu \mathrm{L}$ iTRAQ tag solution (either 114 tag or 117 tag). These solutions were incubated at $37^{\circ} \mathrm{C}$ overnight. The resulting Bcl-2 concentration was $1.4 \times 10^{-7} \mathrm{M}$ which was the same as the reference tag for Bcl-2 in the main manuscript. Subsequently, Bcl-2 digests with two labels were mixed in various volume ratios. MALDI matrix was added and spotted onto a MALDI target plate. The spots were analyzed by using MALDI-TOF/TOF. The summary of the measurements is shown in Table S-1. Figure S-1 shows the peak area ratios derived from our experiments versus the expected amount ratios of $\mathrm{Bcl}-2$ digest. An average $\mathrm{SD}$ of $13.5 \%$ was determined which is in good agreement with Wiese et al. ${ }^{1}$

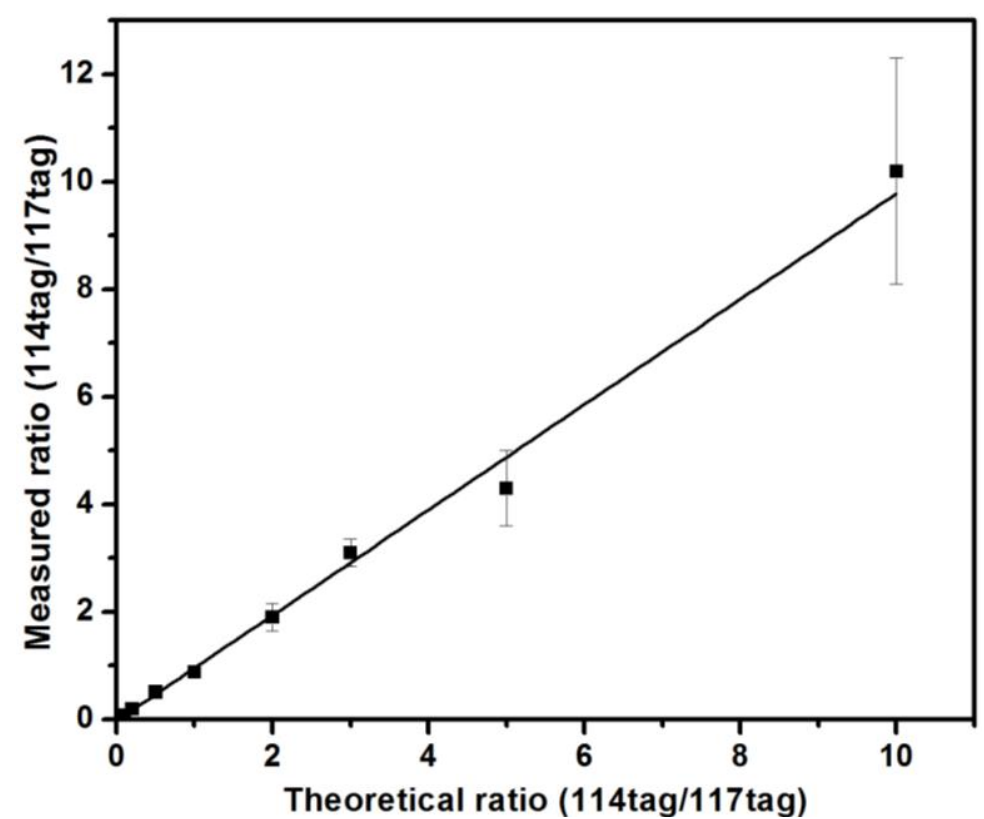

Figure S-1: Linear range between measured peak area ratios of iTRAQ reporter ions $(\mathrm{m} / \mathrm{Z} 114.1$ and $\mathrm{m} / \mathrm{Z} 117.1$ ) and theoretical amount ratios of differentially labeled $B c l-2$ digest with a slope of 0.98(+/-0.054) and $R^{2}$ of 0.9923. An average SD of $17 \%$ was determined (insert: Bcl-2 measurements for different ratios of 114 and 117 tag). 


\section{Sensitivity of iTRAQ quantification strategy with Bcl-2}

The lowest concentration of Bcl-2 which could be quantified by iTRAQ strategy was determined. $1.4 \times 10^{(-5)} \mathrm{M}$ of Bcl-2 was digested and separately labeled with 114 tag and 117 tag by using the procedures described in the above section. The stock concentration of tagged Bcl-2 digest was $1.4 \times 10^{(-6)} \mathrm{M}$. A dilution series of this solution (either 114 tagged or 117 tagged) was made from $1.4 \times 10^{(-6)} \mathrm{M}$ to $1.4 \times 10^{(-9)} \mathrm{M}$. The two tags were mixed in 1:1 ratio. Results are shown below (Table S-2).

\begin{tabular}{|c|c|}
\hline Concentration & $\mathbf{1 1 4 / 1 1 7}$ ratio \\
\hline $1.4 \times 10^{(-6)} \mathrm{M}$ & $0.75(+/-0.26)$ \\
\hline $1.4 \times 10^{(-7)} \mathrm{M}$ & $1.08(+/-0.44)$ \\
\hline $1.4 \times 10^{(-8)} \mathrm{M}$ & $1.21: 1(+/-0.46)$ \\
\hline $1.4 \times 10^{(-9)} \mathrm{M}$ & No peak identified \\
\hline
\end{tabular}

Table S-1: Dilution series for iTRAQ quantification on Bcl-2. The theoretical ratio of two tags was 1:1.

\section{Detection Sensitivity for Bcl-2}

The concentration range for Bcl-2 detection was tested over three orders of magnitude. First, direct detection of digested $\mathrm{Bcl}-2$ was performed. Bcl-2 at three different concentrations (100 nM, $10 \mathrm{nM}, 1 \mathrm{nM}$ ) was digested by trypsin. Digests were detected by MALDI MS and results are shown in Figure S-3. Second, Bcl-2 in different concentrations (100 nM, $10 \mathrm{nM}, 1 \mathrm{nM}$ ) was used for the Bcl-2 immunoassay and digestion experiment on chip (Method details were described in Experimental Section in the main manuscript). Results are shown in Figure S-4. 


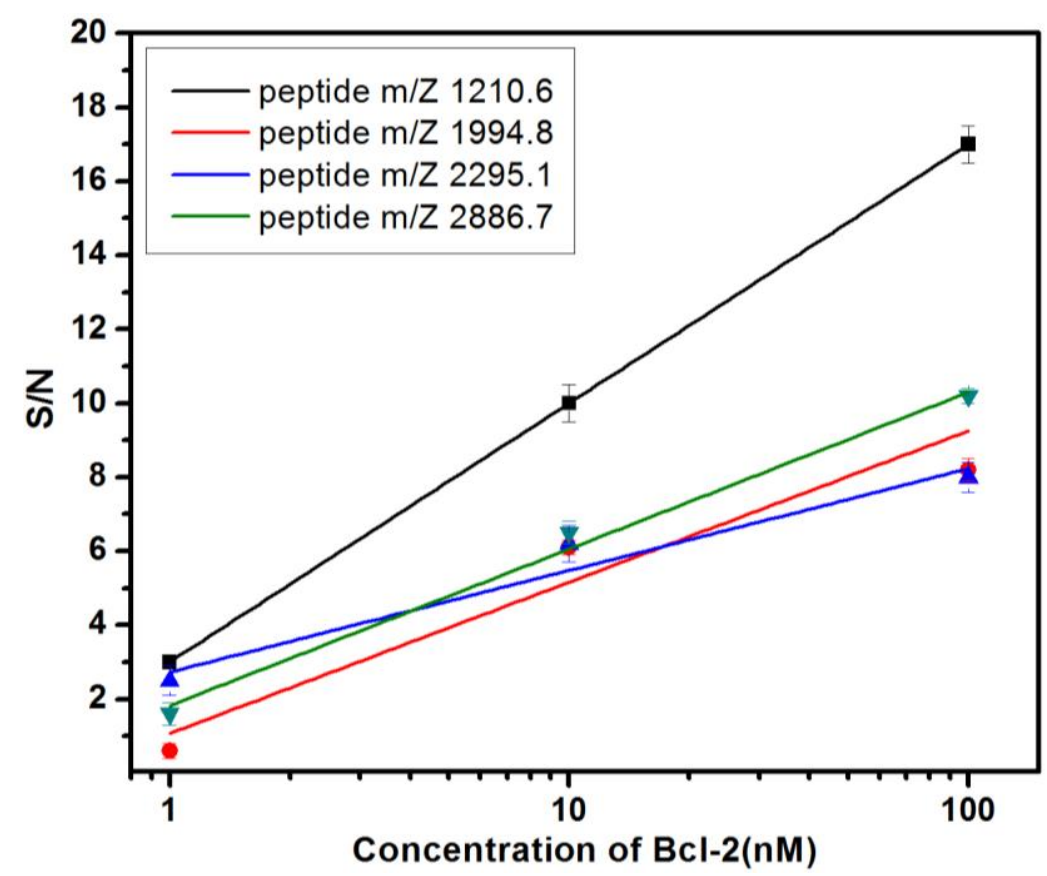

Figure S-2: Signal to noise of Bcl-2 peptides after trypsin digest vs. concentration for various concentrations of Bcl-2.

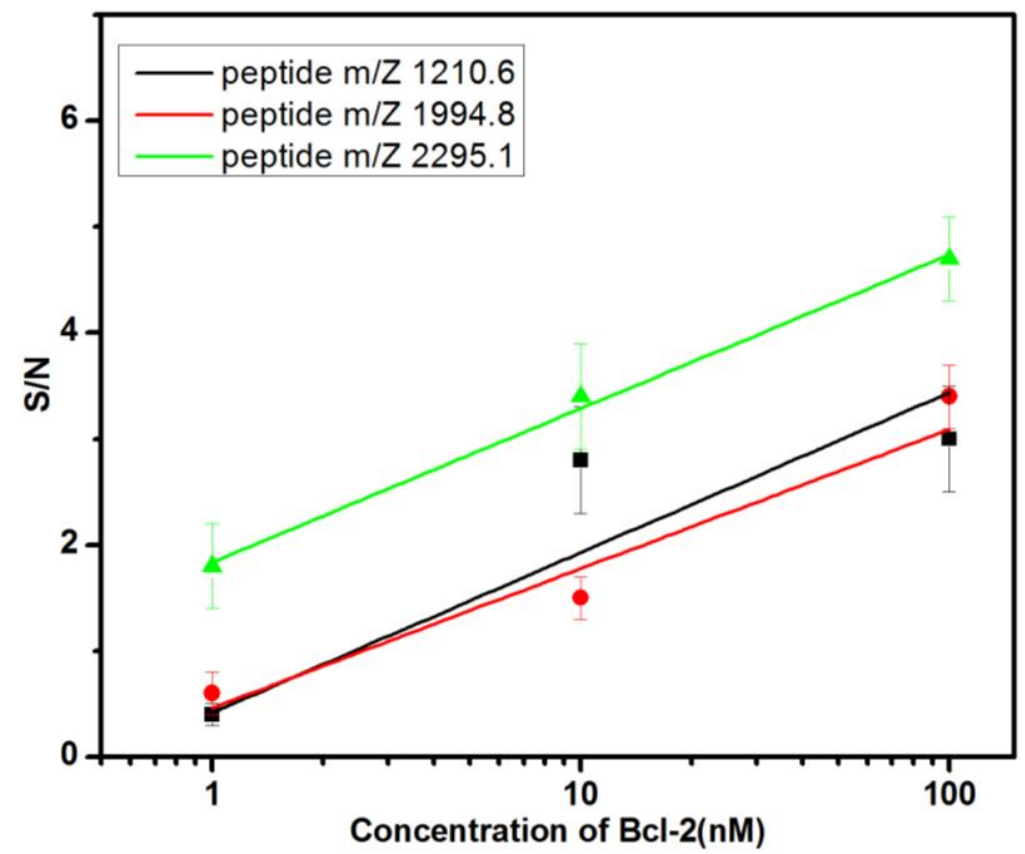

Figure S-3: Signal to noise of Bcl-2 peptides after affinity capture and digestion on chip vs. concentration of Bcl-2. 
Identified Bcl-2 peptides after affinity capture and digestion on-chip

\begin{tabular}{|c|c|c|}
\hline $\begin{array}{c}\text { Mass In the } \\
\text { spectrum }\end{array}$ & $\begin{array}{c}\text { Peptide } \\
\text { Position }\end{array}$ & Sequence \\
\hline 1210.6 & $130-139$ & FATVVEELFR \\
\hline 1950.8 & $111-127$ & DFAEMSSQLHLTPFTAR \\
\hline 1994.8 & $130-146$ & FATVVEELFRDGVNWGR \\
\hline 2295.1 & $165-183$ & EMSPLVDNIALWMTEYLNR \\
\hline 2286.6 & $69-98$ & $\begin{array}{c}\text { TSPLQTPAAPGAAAGPALSP } \\
\text { VPPVVHLTLR }\end{array}$ \\
\hline 3596.8 & $27-63$ & $\begin{array}{c}\text { GYEWDAGDVGAAPPGAPAP } \\
\text { GIFSSQPGHTPHPAASR }\end{array}$ \\
\hline
\end{tabular}

Table S-2: Identification of peaks from $1.4 \mu M$ in the spectrum

\section{References}

(1) Wiese, S.; Reidegeld, K. A.; Meyer, H. E.; Warscheid, B. Proteomics 2007, 7, 340-350. 